OPEN ACCESS

Edited by:

John Greenlee,

University of Utah, United States

Reviewed by:

Olwen C. Murphy,

Johns Hopkins University,

United States

Norio Chihara,

Kobe University, Japan

${ }^{*}$ Correspondence:

Xiaojing $\mathrm{Li}$

lixiaojingfy@163.com

Wen-Xiong Chen

gzchcwx@126.com

tThese authors have contributed equally to this work and share first authorship

Specialty section:

This article was submitted to

Multiple Sclerosis and

Neuroimmunology,

a section of the journa

Frontiers in Neurology

Received: 08 May 2021

Accepted: 26 July 2021

Published: 09 September 2021

Citation:

Chen L, Wu W, Tian Y, Zeng Y, Hou C, Zhu $H$, Zheng $K$, Zhang $Y$, Gao $Y$, Peng $B$, Yang $S$, Wang $X$, Ning $S$, Liao Y, Lin H, Shi K, Li X and Chen W-X (2021) Thyroid Function and Anti-thyroid Antibodies in Pediatric Anti-NMDAR Encephalitis.

Front. Neurol. 12:707046. doi: 10.3389/fneur.2021.707046

\section{Thyroid Function and Anti-thyroid Antibodies in Pediatric Anti-NMDAR Encephalitis}

\author{
Lianfeng Chen ${ }^{\dagger}$, Wenlin $\mathrm{Wu}^{\dagger}$, Yang Tian ${ }^{\dagger}$, Yiru Zeng ${ }^{\dagger}$, Chi Hou, Haixia Zhu, Kelu Zheng, \\ Yani Zhang, Yuanyuan Gao, Bingwei Peng, Sida Yang, Xiuying Wang, Shuyao Ning, \\ Yinting Liao, Haisheng Lin, Kaili Shi, Xiaojing Li* and Wen-Xiong Chen* \\ Department of Neurology, Guangzhou Women and Children's Medical Center, Guangzhou Medical University, Guangzhou, \\ China
}

Objective: Recent studies found that changes of thyroid antibodies (ATAbs), thyroid hormone, and non-thyroidal illness syndrome (NTIS) characterized by thyroid hormone inactivation with low triiodothyronine and high reverse triiodothyronine followed by suppressed thyroid-stimulating hormone (TSH) in adult anti-N-methyl-D-aspartate receptor (NMDAR) encephalitis were associated with disease severity. This study aimed to explore thyroid function and ATAbs in pediatric anti-NMDAR encephalitis and their clinical association.

Methods: We retrospectively analyzed the clinical data of 51 pediatric cases with anti-NMDAR encephalitis hospitalized in Guangzhou Women and Children's Medical Center from August 2016 to 2019.

Results: A percentage of $52.9 \%$ of patients belonged to the ATAb (+) group, with 26 cases both positive for anti-thyroid peroxidase antibodies (TPOAb) and anti-thyroglobulin antibodies (TGAb), and one patient only positive for TPOAb. A percentage of $62.7 \%$ of patients had at least one abnormality in terms of $\mathrm{FT}_{3}$, free thyroxin $\left(\mathrm{FT}_{4}\right)$, or $\mathrm{TSH}$ levels. Meanwhile, $45.1 \%$ of patients were diagnosed with NTIS. Among 25 cases retested for thyroid function 2 months after the initial test, the respectively decreased $\mathrm{FT}_{3}$ and $\mathrm{FT}_{4}$ in 13 and 11 cases on admission returned to normal or closer normal than before; TPOAb in eight cases and TGAb in 12 cases were changed from positivity to negativity. Compared with onset, the level of TPOAb and TGAb at relapse remained stable or significantly decreased, respectively. Compared with the ATAb (-) group, the ATAb (+) group had an older onset age, a higher ratio of movement disorders, elevated rate of sleep disorders, increased anti-nuclear antibody positivity rate, and higher ratio of more than one course of intravenous immunoglobulin treatment. There were no significant differences between the NTIS and non-NTIS groups in clinical characteristics.

Conclusion: Anti-thyroid antibody positivity, abnormality of $\mathrm{FT}_{3}, \mathrm{FT}_{4}$, or $\mathrm{TSH}$ levels and NTIS are frequent in pediatric anti-NMDAR encephalitis. Thyroid antibody and thyroid hormone abnormalities could be improved through the course of treatment of 
anti-NMDAR encephalitis. Cases with ATAbs (+) are at older onset ages and more likely to be treated by intravenous immunoglobulin therapy more than once. Unlike adult antiNMDAR encephalitis, NTIS might not be associated with the clinical characteristics of anti-NMDAR encephalitis in pediatric patients.

Keywords: anti-NMDAR encephalitis, anti-thyroid antibody, thyroid hormone, non-thyroidal illness syndrome, children

\section{INTRODUCTION}

Anti-N-methyl-D-aspartate receptor (NMDAR) encephalitis is an autoimmune disorder associated with autoantibodies binding with the NR1 subunit of the NMDAR receptor (1). The common clinical manifestations of anti-NMDAR encephalitis include psychiatric symptoms, behavioral dysfunction, seizures, movement disorder, speech disorder, cognitive impairment, decreased consciousness, autonomic dysfunction, or central hypoventilation (2). Anti-NMDAR encephalitis can be accompanied with other autoantibodies such as the myelin oligodendrocyte glycoprotein antibody (3), similar to other immune-mediated diseases, presenting more than one immune disorder together (4). Thyroid antibodies are frequently detected not only in patients with autoimmune thyroid disease but also in individuals without overt thyroid dysfunction, including those with rheumatoid arthritis, type 1 diabetes mellitus, Crohn's disease, and neurological disorders such as multiple sclerosis (5). Thyroid hormones are essential in humans. During brain development, thyroid hormones play an important role in the proliferation and differentiation of neuronal and glial progenitors $(6,7)$. In addition, hypothyroidism is associated with a decreased hippocampus size, which is a major area involved in anti-NMDAR encephalitis $(1,8)$. Studies have found that thyroid hormones affect the prognosis of critical and severe diseases, besides substance metabolism, growth, and development (9). Recent studies have reported thyroid antibody (10) and hormone (11) changes in adult anti-NMDAR encephalitis associated with disease severity. Non-thyroidal illness syndrome (NTIS) is characterized by thyroid hormone inactivation, with low triiodothyronine and high reverse triiodothyronine, followed by suppressed thyroid-stimulating hormone (TSH); NTIS is associated with clinical characteristics of adult anti-NMDAR encephalitis (11). However, few such reports are addressing pediatric antiNMDAR encephalitis. In this study, we aimed to evaluate thyroid function and anti-thyroid antibodies in pediatric anti-NMDAR encephalitis.

\footnotetext{
Abbreviations: ATAbs, thyroid antibodies; NMDAR, N-methyl-D-aspartate receptor; ANA, anti-nuclear antibody; CSF, cerebrospinal fluid; EEG, electroencephalograph; $\mathrm{FT}_{3}$, free triiodothyronine; $\mathrm{FT}_{4}$, free thyroxin; IQR, interquartile range; IVIG, intravenous immunoglobulin; IVMP, intravenous methylprednisolone; MRI, brain magnetic resonance imaging; mRS, modified Rankin scale; NTIS, non-thyroidal illness syndrome; SSA, anti-Sjogren syndromerelated antigen A; TGAb, anti-thyroglobulin antibodies; TPOAb, anti-thyroid peroxidase antibodies; TSH, thyroid-stimulating hormone.
}

\section{SUBJECTS AND METHODS}

\section{Subjects}

Children with anti-NMDAR encephalitis were retrospectively recruited from August 2016 to August 2019 in the Department of Neurology of Guangzhou Women and Children's Medical Center. This study was approved by the Ethics Committee of Guangzhou Women and Children's Medical Center. Written and signed consent was obtained from the patients' parents or guardians, who also explicitly consented to publish their personal details, clinical data, and images that could identify them.

\section{Methods \\ Inclusion Criteria}

Patients aged younger than 18 years, diagnosed with antiNMDAR encephalitis according to diagnostic criteria proposed by Graus et al. (2), and undergoing thyroid function tests including free triiodothyronine $\left(\mathrm{FT}_{3}\right)$, free thyroxin $\left(\mathrm{FT}_{4}\right)$, $\mathrm{TSH}$, and anti-thyroid antibody (ATAb) tests including antithyroglobulin antibody (TGAb) and anti-thyroid peroxidase antibody (TPOAb) tests were involved.

\section{Exclusion Criteria}

Patients were excluded if they did not undergo thyroid hormone and thyroid function tests on admission, had known endocrine disorders or severe non-endocrine disorders that may influence thyroid function before anti-NMDAR encephalitis onset, or developed anti-NMDAR encephalitis after viral encephalitis.

\section{Diagnostic Criteria of Hyperthyroidism, Subclinical} Hyperthyroidism, and Non-thyroidal Illness Syndrome Hyperthyroidism was diagnosed by endocrinologists according to the 2016 American Thyroid Association guidelines for diagnosis and management of hyperthyroidism (12). Subclinical hyperthyroidism was defined as a serum TSH concentration below the lower limit of the reference range, with serum $\mathrm{FT}_{4}$ and $\mathrm{FT}_{3}$ concentrations within their reference ranges. NTIS was characterized by the thyroid hormone inactivation with low triiodothyronine and high reverse triiodothyronine followed by suppressed TSH and was diagnosed based on serum $\mathrm{FT}_{3}$ below the age-appropriate normal level and low or normal TSH level (13-18).

\section{Clinical Data Collection}

Clinical data of the involved patients, including age at onset, gender, clinical manifestations, prodromal infection, laboratory test results, electroencephalogram (EEG), brain magnetic resonance imaging (MRI), treatments, outcome, and follow-up, were reviewed. The modified Rankin scale (mRS) was 
used to assess neurological disability at admission and discharge and at the end of follow-up $(3,19,20)$. A poor response was defined as no mRS score improvement or mRS score $\geq 4$ for 4 weeks (21). Relapse was defined as a new onset or worsening of symptoms occurring after at least 2 months of improvement or stabilization. The good long-term prognosis was defined as $\mathrm{mRS}$ score $\leq 2$; the poor long-term prognosis was defined as mRS score $>2(21)$. Cerebrospinal fluid (CSF) pleocytosis was defined as a white blood cell (WBC) count $>15 \times 10^{6} / 1$ in our center. The movement disorders in anti-NMDAR encephalitis include orofacial, limb, or trunk dyskinesias (2).

\section{Laboratory Measurement}

All included patients underwent thyroid function and ATAb examinations upon admission, which were repeated 2 months later in some individuals. Thyroid function indexes, including $\mathrm{FT}_{3}, \mathrm{FT}_{4}$, and TSH levels, and ATAbs (TGAb and TPOAb) were measured using highly sensitive magnetic antibody enzymelinked immunoassays. The normal range for both TPOAb and TGAb was $0-60 \mathrm{IU} / \mathrm{ml}$ in terms of laboratory standards. TPOAb positivity was defined as a TPOAb level higher than $60 \mathrm{IU} / \mathrm{ml}$. TGAb positivity was defined as a TGAb higher than $60 \mathrm{IU} / \mathrm{ml}$. According to ATAb results, a patient with TPOAb or TGAb positivity was assigned to the ATAb-positive group; otherwise, the case was assigned to the ATAb-negative group. The normal ranges of $\mathrm{FT}_{3}, \mathrm{FT}_{4}$, and TSH levels in children vary with age.

NMDAR IgG in both serum and CSF samples was determined by cell-based assays (EUROIMMUN, Lübeck, Germany) and CSF glial fibrillary acidic protein IgG and serum myelin oligodendrocyte glycoprotein IgG. These methods have been reported in detail in our previous study (22).

\section{Treatment}

First-line immunotherapy was performed with intravenous methylprednisolone (IVMP) combined with intravenous immunoglobulin (IVIG) in the acute phase. Two weeks after the first course of IVIG, if patients were not improved well, patients would receive the second course of IVIG treatment or second course of IVMP treatment. Second-line treatment included rituximab or cyclophosphamide administration. In addition, one patient diagnosed with hyperthyroidism was treated with methimazole.

\section{Statistical Analysis}

Statistical analysis was performed with IBM SPSS 20.0 for windows. Quantitative data with normal distribution were described as mean \pm standard deviation and compared by Student's $t$-test or paired-sample $t$-test. Those with skewed distribution were presented as median with interquartile range (IQR) and compared by the Mann-Whitney $U$-test or Wilcoxon signed-rank test. Categorical data were described as frequency and percentage and compared by the chi-square test or Fisher's exact test. $p<0.05$ (two-sided) was considered statistically significant.

\section{RESULTS}

\section{Demographic and Clinical Characteristic Data}

A total of 51 patients (male: female, 21:30) were enrolled, and their clinical features have been reported in our previous study (23). Onset age was $6.3 \pm 2.7$ years, ranging from 1.9 to 15 years. Prodromal infections occurred in 12 cases $(23.5 \%, 12 / 51)$, including respiratory infection (11 cases) and urinary tract infection (1 case). Major symptoms in the whole treatment course were psychiatric symptoms (88.2\%, 45/51), seizures $(84.3 \%, 43 / 51)$, speech disorders $(80.4 \%, 41 / 51)$, sleep disorder $(72.5 \%, 37 / 51)$, abnormal movement $(68.6 \%, 35 / 51)$, fever $(51.0 \%, 26 / 51)$, decreased consciousness $(39.2 \%, 20 / 51)$, and autonomic dysfunction $(5.9 \%, 3 / 51)$. The median initial mRS score evaluated on admission was 4 (IQR, 3-4). One patient diagnosed with hyperthyroidism had emotional lability, heat intolerance, bad temper, profuse sweating, weight loss, and increased appetite, accompanied by second-degree thyroid enlargement without pain on palpation.

\section{Ancillary Test Results CSF Test}

All patients underwent CSF examination after lumbar puncture in the acute phase. WBC count in the CSF at the first lumbar puncture was $44.4 \pm 36.1 \times 10^{6} / 1$, and CSF pleocytosis was observed in $41.2 \%(21 / 51)$ of patients. Protein elevation in the CSF (reference value, $0.15-0.45 \mathrm{~g} / \mathrm{l}$ ) was only detected in $5.9 \%$ (3/51) of patients, with amounts that ranged from 0.53 to 2.09 $\mathrm{g} / \mathrm{l}$. The levels of glucose and chloride were normal except in one patient who showed decreased glucose $(2.35 \mathrm{mmol} / \mathrm{l}$; the ratio of CSF glucose to finger stick glucose was 2.35/4.00 =0.59). In this patient with reduced CSF glucose, CSF WBC was $90 \times$ $10^{6} / 1$ while the CSF protein level was within the normal reference range. These abnormalities of glucose level and WBC count in the CSF returned to normal after treatment with IVIG and IVMP, without antibiotic treatment. The CSF anti-NMDAR antibody was positive in all patients, while serum anti-NMDAR antibody was positive in $41.2 \%(21 / 51)$ of individuals.

\section{ATAbs in Serum and Thyroid Function}

On admission, all patients underwent examination of thyroid antibody tests, including serum TPOAb and TGAb. In total, five patients $(9.8 \%, 5 / 51)$ received IVIG in other hospitals before thyroid antibody tests in our center. Among them, three patients received two courses of IVIG treatment, and two patients received one course of IVIG treatment. The median level of TPOAb and TGAb was $249.9 \mathrm{IU} / \mathrm{ml}$ (IQR, 48.0-454.7 IU/ml) and 78.6 IU/ml (IQR, 15.9-319.8 IU/ml), respectively. Twentyseven cases $(52.9 \%, 27 / 51)$ are TPOAb positive, and their median level of TPOAb was $373.7 \mathrm{IU} / \mathrm{ml}$ (IQR, 246.7-612.6 IU/ml). Twenty-six cases $(51.0 \%, 26 / 51)$ are TGAb positive, of which the median level of $319.8 \mathrm{IU} / \mathrm{ml}$ (IQR, 183.8-499.4 IU/ml) and their TPOAb were positive as well. In total, $52.9 \%$ (27/51) of patients belonged to the ATAb (+) group, and 47.1\% (24/51) were from the ATAb (-) group. 
TABLE 1 | Results of thyroid hormone test on admission.

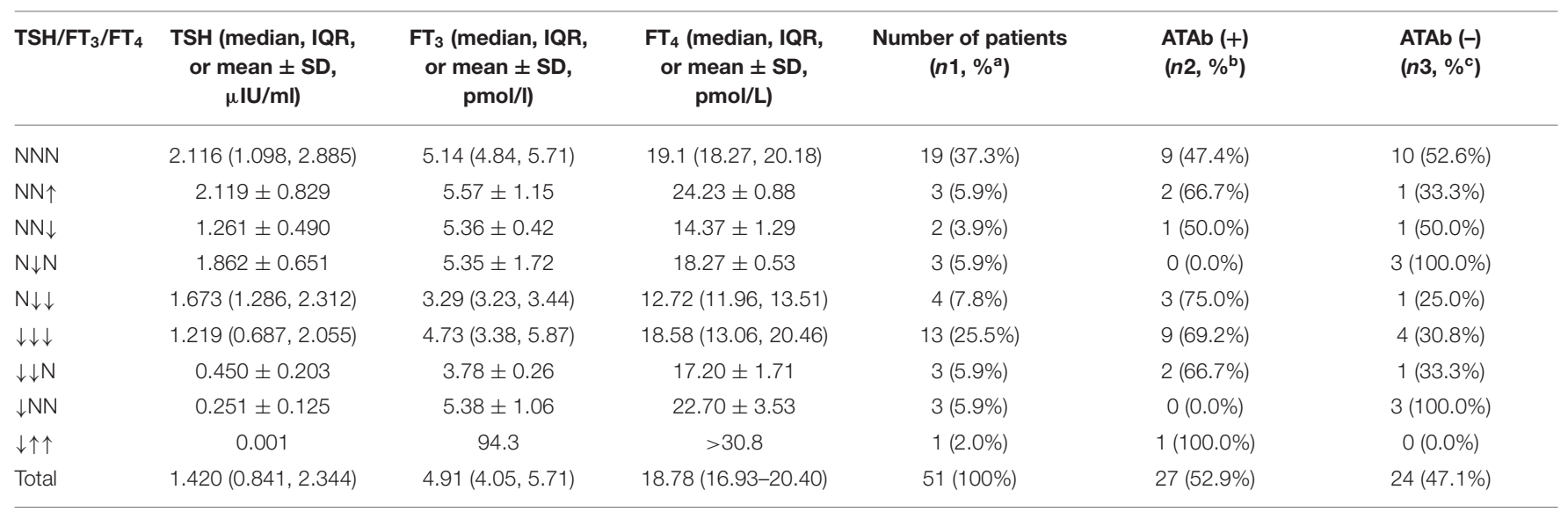

$N$, normal; $\downarrow$, decrease; $\uparrow$, increase; ${ }^{a} n 1 / 51 \times 100 \% ;{ }^{b} n 2 / n 1 \times 100 \% ;{ }^{c} n 3 / n 1 \times 100 \% ; F T_{3}$, free triiodothyronine; $F T_{4}$, free thyroxin; TSH, thyroid-stimulating hormone.

On admission, all included patients underwent examinations for serum $\mathrm{FT}_{3}, \mathrm{FT}_{4}$, and $\mathrm{TSH}$. The median levels of $\mathrm{FT}_{3}, \mathrm{FT}_{4}$, and TSH were $4.91 \mathrm{pmol} / \mathrm{l}$ (IQR, 4.04-5.71 pmol/l), $18.78 \mathrm{pmol} / \mathrm{l}$ (IQR, 16.93-20.40 pmol/l), and $1.420 \mu \mathrm{IU} / \mathrm{ml}$ (IQR, 0.841-2.344 $\mu \mathrm{IU} / \mathrm{ml})$, respectively. Totally, $62.7 \%(32 / 51)$ of patients had at least one abnormality of $\mathrm{FT}_{3}, \mathrm{FT}_{4}$, or $\mathrm{TSH}$ level compared with age-appropriate normal levels. Abnormal $\mathrm{FT}_{3}$ was seen in $47.1 \%$ $(24 / 51)$ of patients, including $\mathrm{FT}_{3}$ reduction $(95.8 \%, 23 / 24)$ and $\mathrm{FT}_{3}$ elevation $(4.2 \%, 1 / 24)$. Abnormal $\mathrm{FT}_{4}$ was found in $45.1 \%$ $(23 / 51)$ of patients, including $\mathrm{FT}_{4}$ decrease $(82.6 \%, 19 / 23)$ and $\mathrm{FT}_{4}$ elevation $(17.4 \%, 4 / 23)$. Decreased TSH as an abnormality was observed in $39.2 \%(20 / 51)$ of patients. Combining $\mathrm{FT}_{3}$, $\mathrm{FT}_{4}$, and TSH to classify these abnormalities (more details in Table 1), patients with concurrently decreased $\mathrm{FT}_{3}, \mathrm{FT}_{4}$, and TSH $(40.6 \%, 13 / 32)$ were the most frequent group, followed by those with decreases in both $\mathrm{FT}_{3}$ and $\mathrm{FT}_{4}$ but normal TSH $(12.5 \%, 4 / 32)$. Decreased TSH and normal $\mathrm{FT}_{3}$ and $\mathrm{FT}_{4}$ levels were only found in three patients $(9.4 \%, 3 / 32)$ who did not show the clinical presentations of hyperthyroidism. However, the thyroid function test in these three patients showed that TSH levels returned to normal at 2 months after the first test; therefore, these three patients did not meet the criteria for diagnosing subclinical hyperthyroidism. Increased $\mathrm{FT}_{3}$ and $\mathrm{FT}_{4}$ amounts and decreased TSH levels were only detected in one patient diagnosed with hyperthyroidism. According to the NTIS diagnosis criteria, $45.1 \%$ (23/51) of patients were diagnosed with NTIS, while 54.9\% (28/51) did not have NTIS. The 28 patients with no NTIS included 19 individuals with normal $\mathrm{FT}_{3}, \mathrm{FT}_{4}$, and TSH levels, three cases with decreased TSH and normal $\mathrm{FT}_{3}$ and $\mathrm{FT}_{4}$ levels, three cases with increased $\mathrm{FT}_{4}$ and normal $\mathrm{FT}_{3}$ and TSH levels, two cases with decreased $\mathrm{FT}_{4}$ and normal $\mathrm{FT}_{3}$ and TSH levels, and the remaining one patient diagnosed with hyperthyroidism.

Two months after the first test, $49.0 \%$ (25/51) of patients were reexamined for thyroid function and ATAbs, including 13 out of 23 cases with initially decreased $\mathrm{FT}_{3}, 11$ out of 19 cases with decreased $\mathrm{FT}_{4}, 18$ out of 27 cases with TPOAb positivity, and 17 out of 26 cases with TGAb positivity. The results showed that $\mathrm{FT}_{3}$ levels in all 13 patients with initially decreased $\mathrm{FT}_{3}$ significantly returned to the normal range or maintained higher than before $(6.1 \pm 1.9 \mathrm{pmol} / \mathrm{l}$ vs. $4.7 \pm 1.4 \mathrm{pmol} / \mathrm{l}$; Mann-Whitney $U$ test, $Z=-2.866, p<0.01$ ); $\mathrm{FT}_{4}$ levels in all 11 patients with initially decreased $\mathrm{FT}_{4}$ were also significantly increased to the normal range $(21.5 \pm 5.3 \mathrm{pmol} / \mathrm{l}$ vs. $18.1 \pm 4.4 \mathrm{pmol} / \mathrm{l}$; MannWhitney $U$-test, $Z=-2.597, p<0.01)$. TPOAb amounts in all 18 patients with initial TPOAb positivity significantly decreased from $446.2 \mathrm{IU} / \mathrm{ml}$ (IQR, 243.6-670.1 IU/ml) to $85.3 \mathrm{IU} / \mathrm{ml}$ (IQR, 42.9-225 IU/ml) (Mann-Whitney $U$-test, $Z=-3.101, p<0.01$ ); TPOAb levels in eight of these 18 patients $(44.4 \%, 8 / 18)$ became negative. TGAb amounts in the 17 patients with initial TGAb positivity significantly decreased from $211.5 \mathrm{IU} / \mathrm{ml}$ (IQR, 146.9$337.3 \mathrm{IU} / \mathrm{ml}$ ) to $24.9 \mathrm{IU} / \mathrm{ml}$ (IQR, 17.3-73.5 IU/ml) (MannWhitney $U$-test, $Z=-2.343, p=0.02)$; TGAb levels in 12 of these 17 patients $(70.6 \%, 12 / 17)$ became negative. Only one patient, diagnosed with hyperthyroidism, had repeated evaluation of ATAbs and thyroid function 4 months after treatment with methimazole, and the results showed that the increased levels of TSH from 0.001 to $0.023 \mu \mathrm{IU} / \mathrm{ml}$, decreased levels of $\mathrm{FT}_{3}$ from $>30.8$ to $7.44 \mathrm{pmol} / \mathrm{l}$, normalized levels of $\mathrm{FT}_{4}$ from $94.3 \mathrm{pmol} / \mathrm{l}$, and decreased levels of TGAb from $>500$ to $393.2 \mathrm{IU} / \mathrm{ml}$ were revealed; TPOAb levels both on admission and reexamination were $>1,300 \mathrm{IU} / \mathrm{ml}$.

\section{Serum Pathogenic Test}

All patients underwent pathogenic serum tests including IgM antibodies for human Chlamydia psittaci, Mycoplasma pneumonia, Legionella pneumonia type 1, Coxiella burnetii, respiratory adenovirus, influenza $\mathrm{A}$, influenza $\mathrm{B}$, parainfluenza, respiratory syncytial virus, and herpes simplex virus. The results showed positive outcomes in the 13 cases $(25.5 \%, 13 / 51)$, including eight cases that are serum Herpes simplex virus IgM positive and five cases that are mycoplasma pneumonia IgM positive, respectively.

\section{Other Coexisting Autoantibodies in Serum and CSF}

Totally, 51 patients underwent a coexisting autoantibody test for serum and CSF samples. Except for anti-NMDAR antibody and ATAbs, the other autoantibodies tested included 
IgG antibodies for glutamate and $\gamma$-aminobutyric acid alpha and beta receptors, alpha-amino-3-hydroxy-5-methyl-4isoxazolepropionic acid receptor1, alpha-amino-3-hydroxy5-methyl-4-isoxazolepropionic acid receptor2, leucine-rich glioma-inactivated 1 protein, and contactin-associated protein-like 2 in CSF and serum, IgG antibodies for myelin oligodendrocyte glycoprotein and aquaporin-4 in serum, and antinuclear antibody, anti-nuclear ribonucleoprotein antibody, anti-Sjogren syndrome-related antigen A (SSA)/52 KD, SSA/60 $\mathrm{KD}$, and anti-Sjogren syndrome-related antigen $\mathrm{B}$ in serum. A percentage of $21.6 \%(11 / 51)$ of patients showed positivity for other autoantibodies. Only anti-nuclear antibody (ANA) positivity was seen in seven cases $(13.7 \%, 7 / 51)$, and only SSA positivity in two cases $(3.9 \%, 2 / 51)$; both ANA and SSA positivities were detected in 2 cases $(3.9 \%, 2 / 51)$. Myelin oligodendrocyte glycoprotein antibody positivity was seen in one patient.

\section{EEG}

All patients underwent EEG. Totally 49 cases had abnormal EEG, including $88.2 \%(45 / 51)$ with slow wave, 35.3\% (18/51) with epileptic activity, and 2.0\% (1/51) with Delta brush.

\section{Imaging Examination}

All patients underwent brain MRI examination in the acute phase, and $15.7 \%(8 / 51)$ had parenchymal lesions, with the most common location being the temporal lobe (five cases), followed by the insular lobe (four cases). Totally three patients underwent ultrasound examination of the thyroid: two cases had a normal organ, while a patient diagnosed with hyperthyroidism showed diffuse goiter and thyroid inferno. All patients underwent tumor screening, including chest computer tomography scans and abdominal and genital MRI scans. No patients had tumors.

\section{Treatment}

All patients were treated by first-line immunotherapy with IVMP combination with IVIG. Thirty-two patients received two courses of IVIG treatment, and 19 patients received only one course of IVIG treatment. Forty-nine patients received one course of IVMP treatment, and only two patients received two courses of IVMP treatment. Totally, 78.4\% (40/51) of patients had a good response to the first-line therapy. The remaining 11 patients had poor response to the first-line immunotherapy, among whom six cases were subsequently treated with rituximab as second-line immunotherapy and improved without relapse; five patients continued treatment with IVIG monthly (a total of $400 \mathrm{mg} / \mathrm{kg} /$ day monthly) and a low oral dose of corticosteroid treatment $(0.5-1 \mathrm{mg} / \mathrm{kg} /$ day of prednisone for $1-2$ weeks, with dosage tapering weekly for 3-6 months). Totally, three out of these five patients, who continued to be treated with IVIG and a low oral dose of corticosteroid, improved without relapse, while the remaining two cases relapsed and were further treated with cyclophosphamide. Consequently, after treatment with cyclophosphamide, these two patients improved without relapse.

In addition, 15 cases were treated with antiepileptic drugs-of these, nine and six patients with two types of antiepileptic drugs for seizures' control needed.
One patient diagnosed with hyperthyroidism was treated with methimazole and a low-iodine diet.

As supportive treatment, one patient received tracheal intubation and mechanical ventilation. Totally three cases accompanied by infection received antibiotic therapy, including meropenem (two cases) and a combination of vancomycin and cefoperazone-sulbactam (one case). In addition, nine cases were treated with risperidone to improve the abnormal mental behaviors.

\section{Course and Relapse}

The median length of the first hospital stay was $28.8 \pm 10.2$ days. Seven patients $(13.7 \%, 7 / 51)$ relapsed during corticosteroid weaning (three cases) or after withdrawal (four cases). Compared with TPOAb levels at onset, TPOAb amounts on relapse tended to decrease without statistical significance [341.8 IU/ml (IQR, 110.6-902.4 IU/ml) at onset vs. $34.6 \mathrm{IU} / \mathrm{ml}$ (IQR, 30.2-145.9 $\mathrm{IU} / \mathrm{ml}$ ) at relapse; Wilcoxon signed-rank test, $p=0.07]$. In addition, compared with TGAb amounts at onset, TGAb levels on relapse were significantly decreased [295.8 IU/ml (IQR, 78.2$482.4 \mathrm{IU} / \mathrm{ml}$ ) at onset vs. $16.6 \mathrm{IU} / \mathrm{ml}(\mathrm{IQR}, 15-23.45 \mathrm{IU} / \mathrm{ml})$ at relapse; paired-sample $t$-test, $p=0.02$ ].

\section{Prognosis}

One patient $(2.0 \%)$ was lost to follow-up for poor compliance. The duration of follow-up was $20.0 \pm 6.7$ months. The median initial mRS score in the 50 followed patients was 4 (IQR, 3-4), which was significantly higher than the value at the last followup of 0 (IQR, $0-1)(Z=-6.386$, Wilcoxon signed-rank test, $p<$ $0.01)$. All the followed-up patients were alive at the last follow-up and achieved a good prognosis $(\mathrm{mRS} \leq 2)$.

\section{Clinical Comparison Between ATAb $(+)$ and ATAb (-) Groups}

There was no significant difference in gender distribution between the ATAb $(+)$ and ATAb $(-)$ groups, whereas onset age was elevated in the ATAb $(+)$ group compared with the ATAb (-) group $(7.1 \pm 2.9$ vs. $5.3 \pm 2.2$ years, $p=0.02)$. As for clinical characteristics, compared with the ATAb (-) group, the ATAb $(+)$ group had higher rates of movement disorders (88.9 vs. $45.8 \%, p<0.01$ ) and sleep disorders (85.2 vs. $58.3 \%, p=0.03$ ), ANA positivity (29.6 vs. $4.1 \%, p=0.03)$, and IVIG treatment $\geq 2$ courses (77.8 vs. $45.8 \%, p=0.02$ ). Details are shown in Table 2.

\section{Clinical Comparison Between Patients With and Without NTIS}

Patients with anti-NMDAR encephalitis were divided into the NTIS and non-NTIS groups based on serum $\mathrm{FT}_{3}$ and TSH amounts. There were no significant differences between these two groups in gender distribution, onset age, clinical symptoms, and rates of prodromal infections, pleocytosis in CSF, ANA positivity, lesions in brain MRI, IVIG treatment $\geq 2$ courses and treatment with rituximab, and $\mathrm{mRS}$ score on admission or at first discharge and relapse rate (more details in Table 3 ). 
TABLE 2 | Comparison of clinical characteristics between the ATAb (+) and ATAb $(-)$ groups.

\begin{tabular}{lccc}
\hline & ATAbs (+) & ATAbs (-) & $\boldsymbol{p}$ \\
& $\boldsymbol{n}=\mathbf{2 7}$ & $\boldsymbol{n}=\mathbf{2 4}$ & \\
\hline Male/female & $12 / 15$ & $9 / 15$ & $0.62^{\mathrm{a}}$ \\
Age (mean \pm SD,years) & $7.1 \pm 2.9$ & $5.3 \pm 2.2$ & $0.02^{\mathrm{b}}$ \\
Fever, $n$ (\%) & $15(55.6)$ & $11(45.8)$ & $0.49^{\mathrm{a}}$ \\
Decrease consciousness, $n$ (\%) & $13(48.1)$ & $7(29.1)$ & $0.49^{\mathrm{a}}$ \\
Movement disorders, $n$ (\%) & $24(88.9)$ & $11(45.8)$ & $<0.01^{\mathrm{a}}$ \\
Epileptic seizures, $n$ (\%) & $22(81.4)$ & $21(87.5)$ & $0.71^{\mathrm{c}}$ \\
Speech disorders, $n(\%)$ & $24(88.9)$ & $17(70.8)$ & $0.16^{\mathrm{c}}$ \\
Psychiatric symptoms, $n$ (\%) & $26(96.3)$ & $19(79.1)$ & $0.09^{\mathrm{c}}$ \\
Sleep disorders, $n$ (\%) & $23(85.2)$ & $14(58.3)$ & $0.03^{\mathrm{a}}$ \\
Prodromal infections, $n$ (\%) & $9(33.3)$ & $3(12.5)$ & $0.08^{\mathrm{a}}$ \\
HSV IgM (+) $n$ (\%) & $4(14.8)$ & $4(16.7)$ & $1.00^{\mathrm{c}}$ \\
Pleocytosis in CSF, $n$ (\%) & $12(44.4)$ & $9(37.5)$ & $0.62^{\mathrm{a}}$ \\
ANA positive, $n$ (\%) & $8(29.6)$ & $1(4.1)$ & $0.03^{\mathrm{c}}$ \\
Lesions in brain MRI, $n(\%)$ & $4(14.8)$ & $4(16.7)$ & $1.00^{\mathrm{c}}$ \\
IVIG ( $\geq 2$ courses), $n$ (\%) & $21(77.8)$ & $11(45.8)$ & $0.02^{\mathrm{a}}$ \\
Rituximab, $n$ (\%) & $4(14.8)$ & $2(8.3)$ & $0.67^{\mathrm{c}}$ \\
Hospital day (d) & $31.0 \pm 9.9$ & $26.1 \pm 9.6$ & $0.06^{\mathrm{d}}$ \\
mRS on admission & $4(3,4)$ & $4(3,4)$ & $0.40^{\mathrm{d}}$ \\
mRS at discharge & $2(2,3)$ & $2(2,3)$ & $0.55^{\mathrm{d}}$ \\
Relapse, $n$ (\%) & $5(18.5)$ & $2(8.3)$ & $0.43^{\mathrm{c}}$ \\
\hline
\end{tabular}

${ }^{a}$ Chi-square test; ${ }^{b}$ independent $t$-test; ${ }^{c}$ Fisher's exact test; ${ }^{d}$ Mann-Whitney U-test. ANA, antinuclear antibody; CSF, cerebrospinal fluid; HSV, Herpes simplex virus; IVIG, intravenous immunoglobulin; MRI, magnetic resonance imaging; mRS, modified Rankin scale; SD, standard deviation.

\section{DISCUSSION}

In the present study, we aimed to evaluate thyroid function and ATAbs in pediatric anti-NMDAR encephalitis. We found that $52.9 \%$ of patients had TPOAb positivity, and $51.0 \%$ had TGAb positivity. Totally $52.9 \%$ of patients belonged to the ATAb $(+)$ group. ATAbs also occur in immune-mediated nervous diseases such as multiple sclerosis (24), optic neuromyelitis $(25,26)$, limbic encephalitis (27), Hashimoto's encephalopathy (28), and adult anti-NMDAR encephalitis (10). ATAbs in adult anti-NMDAR encephalitis were reported in $52.4 \%$ of cases, corroborating the current findings (10). The prevalence rates of ATAbs in a study of adult anti-NMDAR encephalitis and ours were distinctly higher than those of $10-15 \%$ found in the general population $(27,28)$. Besides, we also found that patients in the ATAb $(+)$ group had partly different characteristics than the ATAb (-) group. The ATAb $(+)$ group at older onset age had a higher rate of movement and sleep disorders, had elevated frequencies of ANA positivity, needed more courses of IVIG treatment, and had longer hospital stays. Although in the previous report, the adult patients with antiNMDAR encephalitis in the ATAb (+) group also showed different clinical characteristics from the ATAb (-) group, and those differences were not the same findings in the current work (10). Specifically, the adult patients with anti-NMDAR
TABLE 3 | Comparison of clinical characteristics between NTIS and not NTIS group.

\begin{tabular}{lccc}
\hline & NTIS & Not NTIS & $\boldsymbol{p}$ \\
& $\boldsymbol{n}=\mathbf{2 3}$ & $\boldsymbol{n}=\mathbf{2 8}$ & \\
\hline Male/female & $9 / 14$ & $12 / 16$ & $0.79^{\mathrm{a}}$ \\
Age (mean \pm SD, years) & $6.5 \pm 2.7$ & $6.0 \pm 2.7$ & $0.66^{\mathrm{b}}$ \\
$\quad$ Fever, $n$ (\%) & $9(39.1)$ & $17(60.7)$ & $0.13^{\mathrm{a}}$ \\
Decrease consciousness, $n$ (\%) & $10(43.5)$ & $10(35.7)$ & $0.57^{\mathrm{a}}$ \\
$\quad$ Movement disorders, $n$ (\%) & $15(65.2)$ & $20(71.4)$ & $0.63^{\mathrm{a}}$ \\
Epileptic seizures, $n$ (\%) & $18(78.3)$ & $25(89.3)$ & $0.28^{\mathrm{c}}$ \\
Speech disorders, $n$ (\%) & $18(78.3)$ & $23(82.1)$ & $0.74^{\mathrm{c}}$ \\
Psychiatric symptoms, $n$ (\%) & $21(91.3)$ & $24(85.7)$ & $0.68^{\mathrm{c}}$ \\
$\quad$ Sleep disorders, $n$ (\%) & $16(69.6)$ & $21(75.0)$ & $0.67^{\mathrm{a}}$ \\
Prodromal infections, $n$ (\%) & $6(26.1)$ & $6(21.4)$ & $0.70^{\mathrm{a}}$ \\
Pleocytosis in CSF, $n$ (\%) & $7(30.4)$ & $14(50.0)$ & $0.16^{\mathrm{c}}$ \\
ANA positive, $n$ (\%) & $3(13.0)$ & $6(21.4)$ & $0.48^{\mathrm{c}}$ \\
Lesions in brain MRI, $n$ (\%) & $6(26.1)$ & $2(7.1)$ & $0.12^{\mathrm{c}}$ \\
IVIG ( $\geq 2$ courses), $n$ (\%) & $15(65.2)$ & $17(60.7)$ & $0.95^{\mathrm{a}}$ \\
Rituximab, $n$ (\%) & $3(13.0)$ & $3(10.7)$ & $1.00^{\mathrm{c}}$ \\
Hospital day (d) & $27.3 \pm 10.2$ & $29.8 \pm 9.8$ & $0.32^{\mathrm{d}}$ \\
mRS on admission & $4(3.4)$ & $4(4.4)$ & $0.92^{\mathrm{d}}$ \\
mRS at discharge & $3(2.4)$ & $2(1.3)$ & $0.10^{\mathrm{d}}$ \\
Relapse, $n$ (\%) & $2(8.7)$ & $5(17.9)$ & $0.44^{\mathrm{c}}$ \\
\hline
\end{tabular}

${ }^{a}$ Chi-square test; ${ }^{b}$ independent $t$-test; ${ }^{c}$ Fisher's exact test; ${ }^{d}$ Mann-Whitney U-test. ANA, antinuclear antibody; CSF, cerebrospinal fluid; IVIG, intravenous immunoglobulin; MRI, magnetic resonance imaging; mRS, modified Rankin Scale; SD, standard deviation.

encephalitis in the ATAb $(+)$ group had a higher mRS score at admission or discharge, a higher rate of epileptic seizures and consciousness disorder, and an increased rate of lesions in brain MRI. However, the adult study did not compare the difference in the rates of movement disorders and sleep disorders, ANA positivity, and the course of IVIG treatment between the ATAb (+) and ATAb (-) groups (10). In addition, we also found no differences in the rates of seizures, conscious disorder, and lesions in brain MRI between the ATAb $(+)$ and ATAb (-) groups. The difference between this trial and the adult study might be caused by the difference of study subjects or the limited sample size. Although there were differences between the ATAb $(+)$ and ATAb $(-)$ groups, it is unclear whether ATAbs play a direct pathogenic role in anti-NMDAR encephalitis. It was reported that thyroid globulin might cross-react with myelin-related antigen epitopes in molecular simulation, indicating that TGAb could cause brain injury (24). In addition, TPOAb could specifically bind to cerebellar astrocytes in patients with Hashimoto's encephalopathy (29). It was speculated that TPOAb might affect glial function after interacting with glial cells, thus resulting in neuronal dysfunction (29). However, in this study, ATAbs were not associated with disease severity at onset and the level of TGAb decreased at relapse, and TPOAb remained stable at relapse. We speculated that elevated ATAbs might be associated with increased hyperactivity immune response at the onset of anti-NMDAR encephalitis in the ATAb $(+)$ group, and these 
patients required more courses of IVIG treatment. However, anti-NMDAR encephalitis relapse was not associated with elevated ATAbs in this study.

We found that $62.7 \%$ of patients had at least one abnormality of $\mathrm{FT}_{3}, \mathrm{FT}_{4}$, or $\mathrm{TSH}$ levels compared with age-appropriate normal values. Abnormalities of $\mathrm{FT}_{3}, \mathrm{FT}_{4}$, and TSH were found in $47.1 \%, 45.1 \%$, and $39.2 \%$ of cases, respectively. In addition, $45.1 \%(23 / 51)$ of patients were diagnosed with NTIS, also known as low triiodothyronine syndrome, and could be caused by some pathologies in the absence of thyroid disease (30). NTIS occurs in many hospitalized patients, especially critically ill patients in the $\operatorname{ICU}(31,32)$. NTIS has also been reported in neuroimmune diseases, including anti-NMDAR encephalitis (11,24-26). Studies showed that adult patients with anti-NMDAR encephalitis with NTIS have a higher rate of decreased consciousness, an elevated mRS score at admission, and longer hospital stay (11). However, in this study, no significant differences were found in clinical symptoms, mRS scores at admission or discharge, and hospital stay between the NTIS and non-NTIS groups. The inconsistency between the adult trial and this study may be caused by differences in study subjects. In addition, we could not fully confirm that NTIS had no effects on pediatric anti-NMDAR encephalitis due to the small sample size and only a single time point at admission for the thyroid function tests. NTIS could occur later after hospital admission, so some possible NTIS cases might not have been detected, which might affect the finding that NTIS was not associated with clinical features. Further investigation with large sample size and multiple time point tests for the thyroid function tests is required.

In NTIS, thyroid hormone treatment is controversial. Some researchers believed that proper thyroid hormone therapy is beneficial to disease rehabilitation (30). However, the benefit of thyroid hormone treatment is not always achieved (33, 34). A double-blind randomized placebo-controlled study of children with NTIS after cardiac surgery found that thyroid hormone treatment failed to improve growth and neurodevelopment after long-term observation (33). Meanwhile, others disagreed with thyroid hormone treatment in NTIS patients and considered that NTIS compensatorily responded to diseases and aggressive treatment might be counterproductive (9). The present study did not perform thyroid hormone treatment for NTIS. Nevertheless, $\mathrm{FT}_{3}, \mathrm{FT}_{4}$, or TSH levels returned to normal or significantly increased, accompanied by improved anti-NMDAR encephalitis. Actually, NTIS may be related to variations of inflammatory factors (30). After primary disease improvement, inflammation in the primary disease could be controlled, and NTIS would also be improved. Therefore, we hypothesized that NTIS in pediatric anti-NMDAR encephalitis is a temporary change in an acute phase of the disease, which requires follow-up and can recover without thyroid hormone treatment.

Our study has several limitations. The first limitation is that only a single time point at admission for the thyroid function tests may affect the finding that NTIS was not associated with clinical features. The second limitation is that our study is a retrospective study and lacks follow-up thyroid antibody and thyroid function testing in around half of the subjects. In addition, in terms of the ANA antibody test, ANA positivity was only seen in a total of nine cases, among whom only two patients underwent the concentration of the ANA antibody test (33.32 and 90.29 IU/l, respectively). For the limitation of the retrospectively study, we could not provide the ANA values for the other seven patients.

\section{CONCLUSION}

Anti-thyroid antibody positivity, abnormality of $\mathrm{FT}_{3}, \mathrm{FT}_{4}$, or TSH levels and NTIS are frequent in pediatric antiNMDAR encephalitis. Thyroid antibody and thyroid hormone abnormalities could be improved through the course of treatment of anti-NMDAR encephalitis. Cases with ATAbs (+) at older onset ages are more likely to be treated by intravenous immunoglobulin therapy more than once. Unlike adult antiNMDAR encephalitis, NTIS might not be associated with the clinical characteristics of anti-NMDAR encephalitis in pediatric patients.

\section{DATA AVAILABILITY STATEMENT}

The raw data supporting the conclusions of this article will be made available by the authors, without undue reservation.

\section{ETHICS STATEMENT}

This study was approved by the Ethics Committee of Guangzhou Women and Children's Medical Center (2019052419364384). Written informed consent to participate in this study was provided by the participants' legal guardian/next of kin.

\section{AUTHOR CONTRIBUTIONS}

LC, WW, YT, and YiZ study concept, acquisition of data, and drafting of the manuscript. $\mathrm{CH}, \mathrm{HZ}, \mathrm{KZ}, \mathrm{YaZ}$, and YG study concept and acquisition of data. BP, SY, XW, SN, YL, HL, and KS analysis of data and interpretation. XL and W-XC study concept and critical revision of the manuscript for intellectual content. All the authors gave final approval of the version to be published.

\section{FUNDING}

This present study was supported by the Health and Family Planning Technological Project Foundation of Guangzhou City (Grant No. 20181A011038). The funders had no role in the study concept, study design, data analysis, interpretation, or reporting of the results. The authors had full control of the data and information submitted for publication. 


\section{REFERENCES}

1. Dalmau J. NMDA receptor encephalitis and other antibody-mediated disorders of the synapse: The 2016 Cotzias Lecture. Neurology. (2016) 87:2471-82. doi: 10.1212/WNL.0000000000003414

2. Graus F, Titulaer MJ, Balu R, Benseler S, Bien CG, Cellucci T, et al. A clinical approach to diagnosis of autoimmune encephalitis. Lancet Neurol. (2016) 15:391-404. doi: 10.1016/S1474-4422(15)00401-9

3. Dalmau J, Lancaster E, Martinez-Hernandez E, Rosenfeld MR, Balice-Gordon R. Clinical experience and laboratory investigations in patients with anti-NMDAR encephalitis. Lancet Neurol. (2011) 10:63-74. doi: 10.1016/S1474-4422(10)70253-2

4. Rojas-Villarraga A, Amaya-Amaya J, Rodriguez-Rodriguez A, Mantilla RD, Anaya JM. Introducing polyautoimmunity: secondary autoimmune diseases no longer exist. Autoimmune Dis. (2012) 2012:254319. doi: 10.1155/2012/254319

5. Frohlich E, Wahl R. Thyroid autoimmunity: role of anti-thyroid antibodies in thyroid and extra-thyroidal diseases. Front Immunol. (2017) 8:521. doi: 10.3389/fimmu.2017.00521

6. Desouza LA, Ladiwala U, Daniel SM, Agashe S, Vaidya RA, Vaidya VA. Thyroid hormone regulates hippocampal neurogenesis in the adult rat brain. Mol Cell Neurosci. (2005) 29:414-26. doi: 10.1016/j.mcn.2005.03.010

7. Bernal J. Thyroid hormone receptors in brain development and function. Nat Clin Pract Endocrinol Metab. (2007) 3:249-59. doi: 10.1038/ncpendmet0424

8. Cooke GE, Mullally S, Correia N, O’Mara SM, Gibney J. Hippocampal volume is decreased in adults with hypothyroidism. Thyroid. (2014) 24:43340. doi: 10.1089/thy. 2013.0058

9. Stathatos N, Wartofsky L. The euthyroid sick syndrome: is there a physiologic rationale for thyroid hormone treatment? J Endocrinol Invest. (2003) 26:11749. doi: 10.1007/BF03349153

10. Lin Y, Tan S, Wang Y, Shen X, Shu Y, Shan Y, et al. Anti-thyroid antibodies and thyroid function in anti-N-methyl-d-aspartate receptor encephalitis. Neurochem Int. (2018) 113:107-11. doi: 10.1016/j.neuint.2017.11.019

11. Ma X, Yin Q, Zeng Z, Wang C, Yang Y, Guo S. Thyroid function and autoimmune indications in patients with anti-N-methyl-Daspartate receptor encephalitis. Neuroimmunomodulation. (2018) 25:110-7. doi: 10.1159/000492179

12. Ross DS, Burch HB, Cooper DS, Greenlee MC, Laurberg P, Maia AL, et al. 2016 American Thyroid Association guidelines for diagnosis and management of hyperthyroidism and other causes of thyrotoxicosis. Thyroid. (2016) 26:1343421. doi: 10.1089/thy.2016.0229

13. Jacobs A, Derese I, Vander Perre S, van Puffelen E, Verstraete S, Pauwels L, et al. Non-thyroidal illness syndrome in critically ill children: prognostic value and impact of nutritional management. Thyroid. (2019) 29:48092. doi: 10.1089/thy.2018.0420

14. Van den Berghe G. Non-thyroidal illness in the ICU: a syndrome with different faces. Thyroid. (2014) 24:1456-65. doi: 10.1089/thy.2014.0201

15. Marks SD. Nonthyroidal illness syndrome in children. Endocrine. (2009) 36:355-67. doi: 10.1007/s12020-009-9239-2

16. Warner MH, Beckett GJ. Mechanisms behind the nonthyroidal illness syndrome: an update. J Endocrinol. (2010) 205:1-13. doi: 10.1677/JOE-09-0412

17. Padhi R, Kabi S, Panda BN, Jagati S. Prognostic significance of nonthyroidal illness syndrome in critically ill adult patients with sepsis. Int J Crit Illn Inj Sci. (2018) 8:165-72. doi: 10.4103/IJCIIS.IJCIIS_29_17

18. Ren H, Ren J, Wang G, Hong Z, Gu G, Chen J, et al. The nonthyroidal illness syndrome is associated with postoperative surgical site infections in enterocutaneous fistulae. Int J Surg. (2018) 51:213-7. doi: 10.1016/j.ijsu.2018.02.001

19. Huang Q, Wu Y, Qin R, Wei X, Ma M. Clinical characteristics and outcomes between children and adults with anti-N-Methyl-D-Aspartate receptor encephalitis. J Neurol. (2016) 263:2446-55. doi: 10.1007/s00415-016-8282-1

20. Armangue T, Spatola M, Vlagea A, Mattozzi S, Cárceles-Cordon M, Martinez-Heras E, et al. Frequency, symptoms, risk factors, and outcomes of autoimmune encephalitis after herpes simplex encephalitis: a prospective observational study and retrospective analysis. Lancet Neurol. (2018) 17:76072. doi: 10.1016/S1474-4422(18)30244-8
21. Titulaer MJ, McCracken L, Gabilondo I, Armangué T, Glaser C, Iizuka T, et al. Treatment and prognostic factors for long-term outcome in patients with antiNMDA receptor encephalitis: an observational cohort study. Lancet Neurol. (2013) 12:157-65. doi: 10.1016/S1474-4422(12)70310-1

22. Hou C, Wu W, Tian Y, Zhang Y, Zhu H, Zeng Y, et al. Clinical analysis of antiNMDAR encephalitis combined with MOG antibody in children. Mult Scler Relat Disord. (2020) 42:102018. doi: 10.1016/j.msard.2020.102018

23. Li X, Hou $\mathrm{C}$, Wu WL, Liang $\mathrm{H}$, Zheng $\mathrm{K}$, Zhang $\mathrm{Y}$, et al. Pediatric anti-N-methyl-d-aspartate receptor encephalitis in southern China: analysis of 111 cases. J Neuroimmunol. (2021) 352:577479. doi: 10.1016/j.jneuroim.2021.577479

24. Sakuma R, Fujihara K, Sato N, Mochizuki H, Itoyama Y. Optic-spinal form of multiple sclerosis and anti-thyroid autoantibodies. J Neurol. (1999) 246:44953. doi: 10.1007/s004150050381

25. Wang X, Yi H, Liu J, Li M, Mao ZF, Xu L, et al. Anti-thyroid antibodies and thyroid function in neuromyelitis optica spectrum disorders. J Neurol Sci. (2016) 366:3-7. doi: 10.1016/j.jns.2016.04.039

26. Cho EB, Min JH, Cho HJ, Seok JM, Lee HL, Shin HY, et al. Low T3 syndrome in neuromyelitis optica spectrum disorder: associations with disease activity and disability. J Neurol Sci. (2016) 370:214-8. doi: 10.1016/j.jns.2016.09.039

27. Tüzün E, Erdag E, Durmus H, Brenner T, Türkoglu R, Kürtüncü $M$, et al. Autoantibodies to neuronal surface antigens in thyroid antibodypositive and -negative limbic encephalitis. Neurol India. (2011) 59:4750. doi: 10.4103/0028-3886.76857

28. Schiess N, Pardo CA. Hashimoto's encephalopathy. Ann N Y Acad Sci. (2008) 1142:254-65. doi: 10.1196/annals.1444.018

29. Blanchin S, Coffin C, Viader F, Ruf J, Carayon P, Potier F, et al. Anti-thyroperoxidase antibodies from patients with Hashimoto's encephalopathy bind to cerebellar astrocytes. J Neuroimmunol. (2007) 192:13-20. doi: 10.1016/j.jneuroim.2007.08.012

30. DeGroot LJ. "Non-thyroidal illness syndrome" is functional central hypothyroidism, if severe. hormone replacement is appropriate in light of present knowledge. J Endocrinol Invest. (2003) 26:1163-70. doi: 10.1007/BF03349151

31. Ataoglu HE, Ahbab S, Serez MK, Yamak M, Kayaş D, Canbaz ET, et al. Prognostic significance of high free T4 and low free T3 levels in non-thyroidal illness syndrome. Eur J Intern Med. (2018) 57:915. doi: 10.1016/j.ejim.2018.07.018

32. Langouche L, Jacobs A, Van den Berghe G. Nonthyroidal illness syndrome across the ages. J Endocr Soc. (2019) 3:2313-25. doi: 10.1210/js.2019-00325

33. Mittnacht J, Choukair D, Kneppo C, Brunner R, Parzer P, Gorenflo $\mathrm{M}$, et al. Long-term neurodevelopmental outcome of children treated with tri-iodothyronine after cardiac surgery: follow-up of a double-blind, randomized, placebo-controlled study. Horm Res Paediatr. (2015) 84:1306. doi: 10.1159/000381711

34. Farwell AP. Nonthyroidal illness syndrome. Curr Opin Endocrinol Diabetes Obes. (2013) 20:478-84. doi: 10.1097/01.med.0000433069.0 9294.e8

Conflict of Interest: The authors declare that the research was conducted in the absence of any commercial or financial relationships that could be construed as a potential conflict of interest.

Publisher's Note: All claims expressed in this article are solely those of the authors and do not necessarily represent those of their affiliated organizations, or those of the publisher, the editors and the reviewers. Any product that may be evaluated in this article, or claim that may be made by its manufacturer, is not guaranteed or endorsed by the publisher.

Copyright (C) 2021 Chen, Wu, Tian, Zeng, Hou, Zhu, Zheng, Zhang, Gao, Peng, Yang, Wang, Ning, Liao, Lin, Shi, Li and Chen. This is an open-access article distributed under the terms of the Creative Commons Attribution License (CC BY). The use, distribution or reproduction in other forums is permitted, provided the original author(s) and the copyright owner(s) are credited and that the original publication in this journal is cited, in accordance with accepted academic practice. No use, distribution or reproduction is permitted which does not comply with these terms. 